\title{
El empoderamiento productivo de la mujer como consecuencia de la Inclusión Financiera
}

\section{The productive empowerment of women as a result of Financial Inclusion}

\author{
Andrea Navas $\mathrm{R}^{1}$. \\ Universidad Internacional del Ecuador, Ecuador \\ Jorge Moncayo L. \\ Investigador independiente, Ecuador
}

Autor por correspondencia: manavasre@uide.edu.ec; moncayo_jorge@ hotmail.com

Fecha de recepción: 07 de agosto del 2019 - Fecha de aceptación: 04 de diciembre del 2019

\section{Resumen}

El documento analiza la importancia de la inclusión financiera y la relación que genera con el desarrollo económico de la mujer a través del emprendimiento productivo. El objetivo es mostrar la importancia que produce el trabajo mancomunado entre los sectores que promuevan el acceso a servicios financieros y la creación de emprendimientos para buscar la igualdad de género, para esto se señala la rentabilidad que provocan las mujeres, la evolución de la creación de empresas y sus fuentes de financiamiento, las barreras que se enfrentan al momento de iniciar los emprendimientos y los beneficios que conlleva la inclusión financiera. Por último, los autores resaltan las buenas prácticas y políticas públicas que fomentan los objetivos de igualdad y crecimiento económico de las mujeres.

Palabras clave: Emprendimiento; inclusión financiera; mujer; empoderamiento

\begin{abstract}
The document analyzes the importance of financial inclusion and the relationship it generates with the economic development of women through productive entrepreneurship. The objective is to show the importance of access to financial services and the creation of ventures to seek gender equality, for this the profitability caused by women, the evolution of the creation of companies and their sources of financing, the barriers that are faced when initiating the ventures and the benefits that financial inclusion entails. Finally, the authors highlight good practices and public policies that promote the goals of equality and economic growth for women.

Key words: Entrepreneurship; finance inclusion; women; empowerment
\end{abstract}

\footnotetext{
${ }^{1}$ Las opiniones expresadas en este documento son las de los autores y no deben atribuirse a ninguna institución que pudieran estar asociados.
} 


\section{Introducción}

"La inclusión financiera se refiere al acceso que tienen las personas y las empresas a una variedad de productos y servicios financieros útiles y asequibles que satisfacen sus necesidades — como pagos y transferencias, ahorro, seguros y crédito - y que son prestados de una manera responsable y sostenible.” (Banco Mundial , 2018)

Si bien en el mundo entero aún existen brechas de acceso a crédito según el nivel de ingresos y nivel de educación, también lo hace en función del género, perjudicando a las mujeres. La intención de generar un emprendimiento por parte de mujeres es menor a la de hombres a nivel mundial y esto debido a que existe una percepción por parte del género femenino en una menor capacidad en autosuficiencia, finanzas y tecnología. Aumentado las barreras para la inclusión y el desarrollo económico de las mujeres.

El objetivo de este estudio es analizar la realidad de la inclusión financiera en zonas subdesarrolladas y relacionarlas con el empoderamiento principalmente femenino para compararlo paso a paso con la situación ecuatoriana.

\section{Metodología}

La metodología utilizada será a través de un análisis cualitativo, utilizando la observación documental como técnica para recopilar información como un proceso analítico, crítico y estructurado, en relación a: inclusión financiera, las mujeres relacionadas a la inclusión financiera y los emprendimientos femeninos en los estratos más bajos, para así, poder generar las relaciones y comparaciones pertinentes para describir e interpretar la relación de dicha situación social como parte de un mismo tema.

Se utilizará el método deductivo para que, sobre la base de conclusiones generales de estudios realizados a nivel mundial en países subdesarrollados, obtener explicaciones y propuestas aterrizadas a Ecuador.

\section{Inclusión Financiera}

Entre los siglos XVIII y XIX surgen las primeras entidades financieras con naturaleza social y un enfoque hacia el fomento de acceso financiero tanto para crédito como para ahorro a personas de bajos recursos y pequeños empresarios, a través de cooperativas, cajas de ahorro, uniones de crédito y otras.

La inclusión financiera se refiere al acceso que tienen las personas y las empresas a una variedad de productos y servicios financieros útiles y asequibles que satisfacen sus necesidades - como pagos y transferencias, ahorro, seguros y crédito - y que son prestados de una manera responsable y sostenible (Banco Mundial , 2018).

Es a principios del año 2000 cuando el objetivo de incluir a segmentos no atendidos por el sistema bancario tradicional comienza a formar parte de las agendas gubernamentales, es entonces en el 2003 cuando nace el concepto de Inclusión Financiera; para en el año 2009 crear la Alianza para la Inclusión Financiera (Roa, 2013) quienes buscan cambiar el entorno con 
menores costos, mayor proximidad a puntos de acceso de servicios financieros, menores requisitos para la apertura de una cuenta, políticas gubernamentales destinadas a promover la inclusión como cuentas básicas, el pago de bonos y subsidios a través de acreditaciones a cuentas en una entidad financiera (Allen, Demirgug- Kunt, Klapper, \& Martinez, 2018) para lo cual es importante sea considerado también el incentivo al desarrollo de innovaciones tecnológicas enfocadas a conocer las necesidades de los consumidores, regulaciones dinámicas que velen por la protección de la información, y sobre todo lograr compromisos y acuerdos entre el sector público y privado para buscar objetivos comunes.

Entre las barreras de mayor recurrencia por las cuales las personas se encuentran excluidas de los servicios financieros están: no tener el dinero suficiente, altos costos, largas distancias a los puntos de accesos, malas experiencias, documentación requerida y temas culturales o religiosos (Banco Mundial , 2018).

\section{Las mujeres como sector financiero rentable}

Si bien en el mundo entero aún existen brechas de acceso a crédito según el nivel de ingresos y nivel de educación, también lo hace en función del género, perjudicando a las mujeres como se puede notar en el siguiente gráfico.

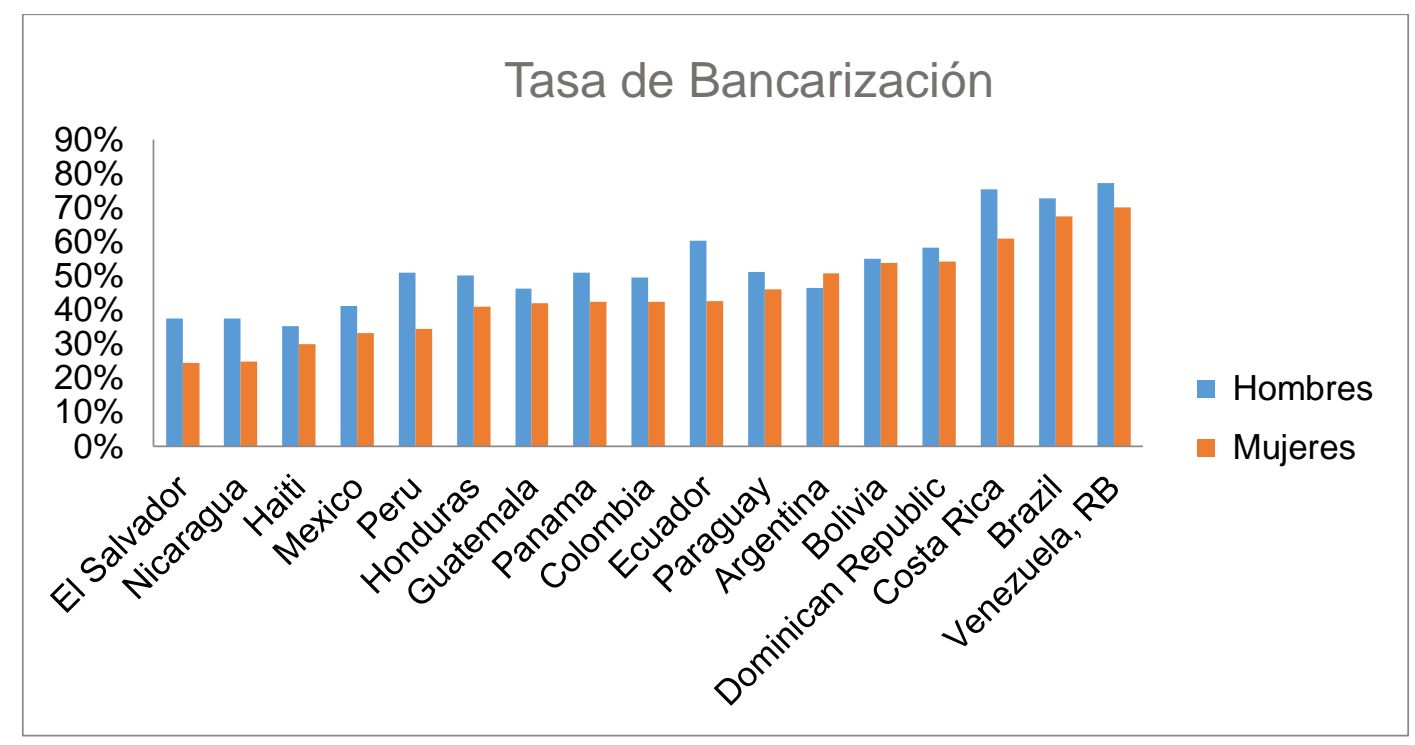

Figura No. 1: Tasa de Bancarización en la Región

Tomado de: Banco Mundial, Global Findex 2017

A nivel mundial tan solo el 58\% de mujeres tiene cuenta en una institución financiera formal, mientras que para los hombres el porcentaje es de 65\%. Para América Latina y el Caribe los valores van de $49 \%$ de acceso a mujeres y $54 \%$ para hombres (Holloway, Niazi, \& Rouse, 2018). En general, en los países en desarrollo existe una desigualdad de 9 puntos porcentuales promedio entre mujeres y hombres en cuanto a tener una cuenta en una institución financiera formal (Banco Mundial , 2018). Sin embargo, esta no es la única diferencia por género en términos financieros; según investigaciones de (CEPAL, 2018, pág. 3), se puede observar que aún existen divergencias en cuanto a la diferencia de género en relación de ingresos propios, es así que "Más de la mitad de las mujeres de la región de América del Sur y el Caribe, o no tienen 
ingresos propios (una de cada tres), o sus ingresos son menores a un salario mínimo (una de cada cuatro)". Así también, la brecha en el salario entre mujeres y hombres es en promedio alrededor de $25 \%$ menos para el género femenino.

Si bien Ecuador está clasificado como un país de renta media, las brechas de género persisten: Las mujeres ganan hasta un $25 \%$ menos que sus pares hombres por trabajos iguales: a) Trabajan entre 16 y 23 horas (en promedio) más que los hombres cada semana. b) No tienen tiempo para invertir en educación, trabajo remunerado, emprendimientos o recreación, como resultado de esto en el ámbito rural el 15,2\% de las mujeres son analfabetas; muchas son pobres de tiempo y no cuentan con recursos propios. c) El $42.6 \%$ de la población ocupada en la producción agrícola son mujeres. Estas actividades, por lo general, representan la principal fuente de recursos de las familias (ONU MUJERES, 2018).

Se evidencia que el respaldo masculino en términos financieros aún es indispensable en la región, muchas mujeres dependen económicamente de sus maridos y una de cada tres mujeres casadas no tienen control sobre el gasto en su hogar. En 15 países las esposas requieren contar con una autorización de sus esposos para poder trabajar (Banco Mundial , 2018). Estereotipos identifican al hombre como mayormente vinculado al mundo financiero y con un mejor criterio en cuanto a dinero e inversión, en tal razón, entre las principales barreras para que mujeres accedan a crédito se encuentran; su situación conyugal, y garantías por medio de bienes inmuebles (CEPAL, 2018). Sin embargo, según un estudio realizado por (Holloway, Niazi, \& Rouse, 2018) existen otras barreras como:

- Del lado de la demanda:

- Falta de poder de negociación dentro del hogar.

- Demanda de tiempo femenino relacionado con labores domésticas.

- Falta de identificación formal.

- Movilidad reducida.

○ Menores porcentajes de propiedad de teléfonos móviles entre las mujeres necesarios en acceso a productos digitales.

- Del lado de la Oferta:

- Productos inadecuados.

- Falta de políticas y prácticas específicas al género.

- Canales de distribución inadecuados.

- Barreras jurídicas:

- Desventajas para mujeres en requisitos para apertura de cuenta.

- Obstáculos a la identificación formal.

- Impedimentos jurídicos para poseer y heredar bienes para dar una garantía.

- Falta de sistemas de información crediticia igualitaria.

Son entonces dichos factores los limitantes para lograr empoderamiento productivo femenino generando una condición complicada en el acceso a recursos de inversión para la generación de emprendimientos, lo que sin duda demuestra que el mercado femenino es muy interesante para la generación de productos financieros específicamente generados para mujeres, 
o para una regularización en las oportunidades que ellas tienen al momento de entrar en el sistema financiero.

\section{Empoderamiento y Emprendimiento femenino}

Entre las estrategias propuestas por la (ONU MUJERES, 2018) está como número 6; "Contener los efectos adversos de la desaceleración económica en la igualdad de género. Entre las acciones propuestas para esta estrategia se menciona: "Proteger el gasto social con efectos positivos en la igualdad de género y el empoderamiento económico de las mujeres", "Incorporar el enfoque de género en los programas dirigidos a administrar, reducir y prevenir la deuda." Para esto es importante se logre conceptualizar el término empoderamiento, el cual está concebido como el proceso que promueve el aumento de capacidades de individuos o grupos para que tomen decisiones (Vazquez, Camacho, \& Garcia, 2016). El tema del empoderamiento femenino ha sido percibido durante la última década como una puerta hacia la equidad de género, por lo cual, varios organismos internacionales en concordancia con el cumplimiento del tercer Objetivo del Milenio de 2015, sobre: "Igualdad de oportunidades para el Hombre y la mujer", generaron una serie de estrategias y contenidos en éste ámbito. Sin embargo, a pesar de las acciones generadas hasta ese año, no se logró mitigar por completo dicha desigualdad, es así que la nueva Agenda 2030 para el Desarrollo Sostenible (Naciones Unidas, 2018), en su punto No 27 menciona:

Procuraremos sentar unas bases económicas sólidas en todos nuestros países. El crecimiento económico sostenido, inclusivo y sostenible es esencial para lograr la prosperidad, lo que solo será posible si se comparte la riqueza y se combate la desigualdad de los ingresos. Trabajaremos para construir economías dinámicas, sostenibles, innovadoras y centradas en las personas, promoviendo en particular el empleo de los jóvenes y el empoderamiento económico de las mujeres, así como el trabajo decente para todos [...] (Naciones Unidas, 2018, pág. 9)

Durante los últimos años se han generado una serie de programas que buscan fomentar por parte de las mujeres el emprendimiento y la gestión de riesgo, valorando factores como su maternidad y su alto nivel de responsabilidad. Es también sobre esta base que se promueve la posibilidad de que las mujeres se conviertan en un segmento específico atractivo para participar del sistema financiero, mitigando una serie de barreras para el acceso a un crédito productivo femenino. Según estudios realizados, (Holloway, Niazi, \& Rouse, 2018), para conocer el efecto de ofrecer cuentas de fácil acceso a mujeres cabeza de familia, más del $80 \%$ aprovecharon esta oportunidad y abrieron su cuenta, adicional, se evidenció que aumentaron sus gastos en términos de educación, comida, entretenimiento; por lo tanto, mejoró el nivel de escolaridad de sus hijos y se tuvo mejor respuesta en emergencias de salud, esto ya que durante las últimas décadas, el rol de la mujer ha tenido una transformación escalando su posición social en distintas áreas (Amador- Ruiz \& Briones- Peñalver, 2017) logrando así que entre el 2000 y 2010, el crecimiento en los ingresos de mujeres de América Latina y el Caribe contribuyó a reducir en un 30\% la pobreza extrema (BID, 2018 ). La participación fémina en comunidades rurales pobres fomenta un ambiente de trabajo cooperativo y humano convirtiendo al emprendimiento en la mejor estrategia para lograr el empoderamiento de mujeres (Vazquez, Camacho, \& Garcia, 2016) quienes, aunque parezca contradictorio, representan un $64 \%$ de las decisiones sobre consumo, 
sobre esto las mujeres efectúan o inciden en hasta el $80 \%$ de las decisiones de compra, lo que incluye desde electrodomésticos, automóviles o servicios médicos (BID, 2018 ).

El empoderamiento femenino es reforzado por medio de las innovaciones sociales lo cual aumenta la productividad y el crecimiento en ámbitos sociales (Vazquez, Camacho, \& Garcia, 2016), es así que las mujeres podrían ser grandes catalizadoras del progreso de un país, principalmente por su especial enfoque hacia la familia, mejorando sus niveles de educación, nutrición y salud, contribuyendo al desarrollo de capital humano, al crecimiento sostenible e inclusivo y, por ende, ayudando a reducir la pobreza (Fundación de Microfinanzas BBVA, 2018). Esta información debe ser vista como una oportunidad para instituciones financieras y generadores de políticas públicas, para lograr el enfoque correcto de los créditos, así como el debido seguimiento de los mismos hacia un sector que fomente condiciones de mejora para la sociedad, sobre todo en los estratos más bajos.

Si bien la antes mencionada disparidad de género ha hecho que aún las mujeres practiquen agricultura en terrenos más reducidos y menos productivos (Banco Mundial , 2018), así como ser propietarias de negocios más pequeños, los resultados que se generan en dichos ámbitos pueden llegar a tener un mayor impacto no solo en su economía sino incluso en la calidad de vida de un país, esto ya que la utilización de sus ganancias son destinadas de manera más responsable pensando en el bienestar familiar, por lo que puede decirse que existe una correlación positiva entre el nivel de empoderamiento productivo femenino y la calidad de vida de un país teniendo además un impacto cíclico. Es por tanto imprescindible lograr cambiar ciertos paradigmas ya que la intención de generar un emprendimiento por parte de mujeres es menor a la de hombres a nivel mundial y esto debido a que existe una percepción por parte del género femenino en una menor capacidad en autosuficiencia, finanzas y tecnología. Así también, según estudios se determina que el abandono por parte de las mujeres a sus actividades empresariales es mayor debido a: responsabilidades familiares, dificultad de capital humano y financiero y, la desigualdad que existe entre hombres y mujeres frente al riesgo (Garcia, Jimenez, \& Escamilla, 2018). Sin embargo, es interesante observar que aquellas emprendedoras femeninas que tomaron la decisión de empezar su negocio, cuentan con habilidades más fuertes en cuanto a gerencia, lo que hace que tengan un mayor entendimiento del negocio y por lo tanto mejores resultados en negociación con sus respectivos stakeholders (Dastourian, Kawamorita, Seyyed Amiri, \& Moradi, 2017).

Aunque la proporción de mujeres sin acceso a ingresos propios es mucho menor que décadas pasadas, aún existe una brecha en relación con los hombres del mismo sector socioeconómico, en contextos de recurrente discriminación laboral, segregación ocupacional y brechas salariales, por lo que, sin un acompañamiento de políticas públicas favorables, su empoderamiento económico continuará incompleto (ONU MUJERES, 2018). En este sentido en Ecuador se ha optado como política pública que promueva el emprendimiento femenino en sectores socioeconómicos bajos, por medio de la generación del Crédito de Desarrollo Humano (CDH). El Ministerio de Economía Popular y solidaria, en el marco de su programa Bono de desarrollo Humano (BDH), busca generar apoyo económico mensual específicamente a las personas en extrema pobreza, con la intención de que este bono logre fomentar el emprendimiento ha generado el Crédito de Desarrollo Humano (CDH), el cual es el adelanto acumulado del BDH por 1 o 2 años, mismo que se entrega principalmente para fomentar 
actividades productivas específicamente de mujeres con extrema pobreza, quienes gracias a este pudieron abrir su emprendimiento (MIES, 2018). A pesar de aquello, este tipo de políticas no son suficientes ya que según datos del GEM 2017, en Ecuador, el principal factor que restringe el emprendimiento en comparación con el promedio mundial, son justamente las políticas gubernamentales y el apoyo financiero, en este, la falta de productos dedicados a emprendedores y la poca cultura de inversión ángel, así como la ausencia de mercado de valores son sus principales limitantes (Lasio, Ordeñana, Caicedo, Samaniego, \& Izquierdo, 2017).

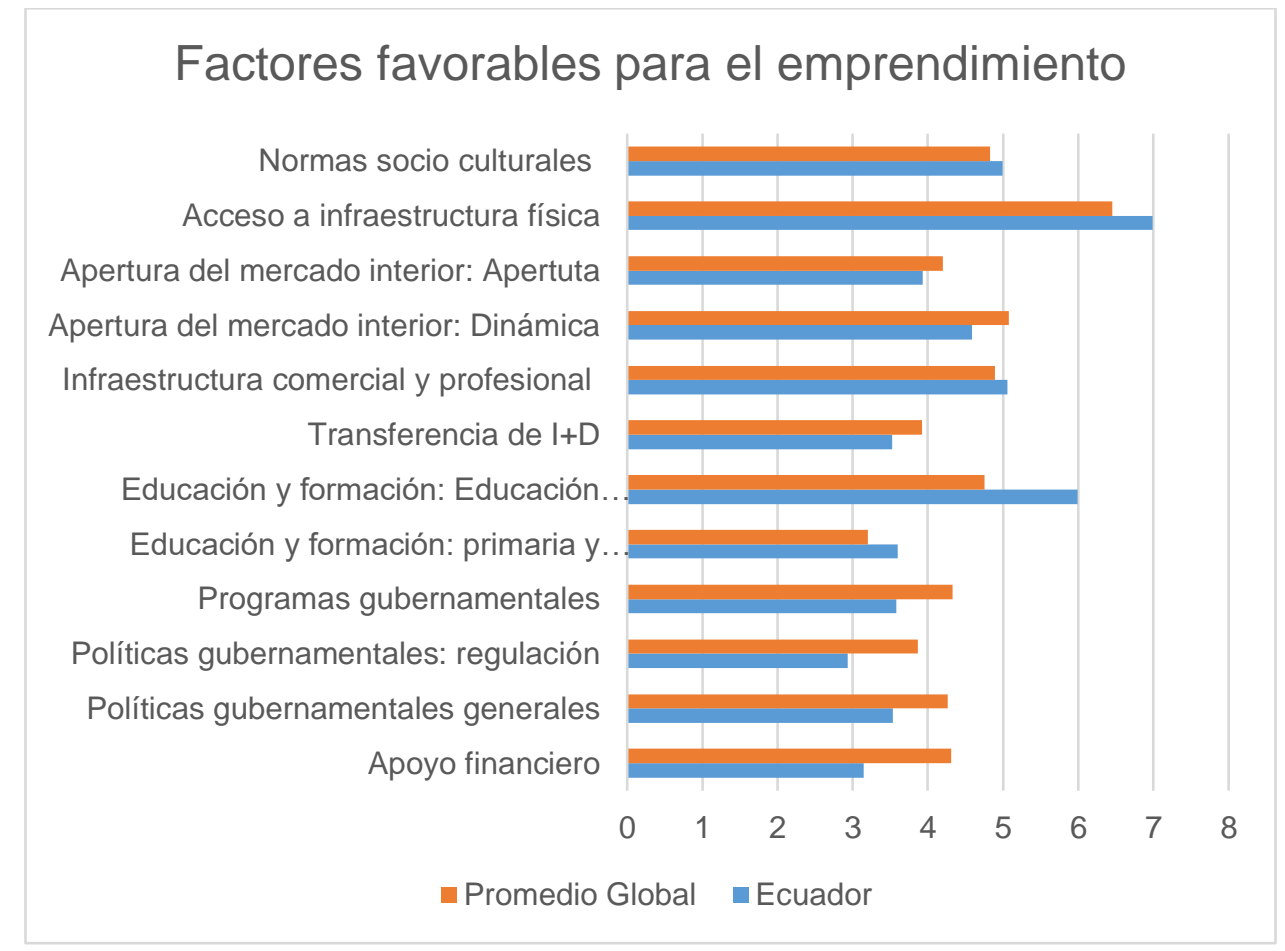

Figura No. 2: Factores clave para el emprendimiento Tomado de: Global Entrepreneurship Ecuador 2017

Los procesos de apoyo por parte del gobierno hacia personas de bajos recursos son usualmente realizados a través de tangibles, o ayudas económicas; son muy pocas las veces que en dicho apoyo se incluye capacitaciones para mejorar el nivel y productividad en emprendimientos (Moreno, 2015), sin embargo, cada vez son más los estudios que señalan la importancia de promover y fomentar un espíritu emprendedor en las personas. En este sentido, se han identificado tres elementos básicos de instrucción para impulsar y fortalecer el emprendimiento, los cuales son: conocimiento, habilidades y por último identificación de atributos personales como: la actitud hacia el entorno y las percepciones de oportunidades, donde, cabe mencionar, las mujeres han tenido un mejor resultado. No obstante, las emprendedoras de bajos recursos usualmente tienen una falta abismal en cuanto a los conocimientos necesarios para desarrollar sus negocios de manera óptima, lo cual repercute en el momento de enfrentar desafíos de mercado, tecnología o globalización, por lo que aprender a enfrentar estos retos les permitiría alcanzar una competitividad sostenible (Amador- Ruiz \& Briones- Peñalver, 2017). 
Sobre los datos recabados por la investigación sobre encuestas de (Garcia, Jimenez, \& Escamilla, 2018), si bien la intención de emprender en mujeres es de un 35\% versus el $30 \%$ en hombres, la oportunidad de hacerlo es de $72 \%$ para hombres y tan solo de $28 \%$ para mujeres. El mismo estudio menciona que aquellas mujeres que han emprendido, lo han hecho mayormente en actividades de servicios con más de un $80 \%$. En Ecuador las proporciones de la Actividad emprendedora temprana (TEA) entre hombres y mujeres son similares, el porcentaje de mujeres emprendedoras es de 52\% versus el $48 \%$ de hombres emprendedores, naciendo un $66 \%$ en áreas urbanas y un $34 \%$ en áreas rurales (Lasio, Ordeñana, Caicedo, Samaniego, \& Izquierdo, 2017). Datos que durante los últimos años han sido crecientes en términos del género femenino, en parte gracias al apoyo que se comienza a dar a este sector como se mencionó antes a través por ejemplo a través del CDH.

Los emprendedores son producto de su entorno social por lo que emprender es entonces un acto social (Dastourian, Kawamorita, Seyyed Amiri, \& Moradi, 2017) que genera beneficios no solo para el emprendedor desde su sentido salarial, sino que atribuye soluciones a problemáticas identificadas en su entorno. Un mayor empoderamiento financiero lleva a reducir la pobreza y las brechas en los ingresos, teniendo su mayor impacto en los hogares más pobres (San Vicente Portes, Atal, \& Juárez, 2019).

En 2010, ONU Mujeres difundió los Principios para el Empoderamiento de las Mujeres:

Establecer un liderazgo empresarial de alto nivel que promueva la igualdad de género. 2 . Tratar a todas las mujeres y los hombres de manera justa en el entorno de trabajo, lo cual implica el respeto y la promoción de los derechos humanos y la no discriminación. 3. Asegurar la salud, seguridad y bienestar de todas las trabajadoras y los trabajadores. 4. Promover la educación, la formación y el desarrollo profesional de las mujeres. 5. Implementar un desarrollo empresarial, cadenas de suministro y prácticas de marketing que empoderen a las mujeres. 6. Promover la igualdad a través de iniciativas de la comunidad e incidencia. 7. Evaluar e informar públicamente los avances de las medidas a favor de la igualdad de género (BID, 2018 ).

Es importante señalar que en el caso del antes mencionado $\mathrm{CDH}$, en base de las cifras manejadas por el ministerio a cargo de este, se señala que el $70 \%$ del dinero entregado en crédito con fines de apoyo a emprendimientos fue utilizado para consumo y necesidades emergentes del hogar por parte de las beneficiarias (MIES, 2018) lo que permite inferir la importancia de un acompañamiento de los entes prestadores del dinero en términos de un correcto uso del mismo para lograr un crecimiento de los emprendimientos así como su sostenibilidad.

\section{Beneficios de la inclusión financiera de la mujer emprendedora}

Los argumentos más fuertes para la inclusión financiera de la mujer son económicos. El acceso a los sistemas financieros formales permite aumentar la productividad, lo que está directamente vinculado con el desarrollo económico. Una mayor inclusión de las mujeres puede aumentar las ganancias entre 2\% al 3.5 \% del GDP (International Finance Corporation, 2018). Debido a que incrementar el acceso a financiamiento mejora la oportunidad del mercado laboral, así como el crecimiento de las micro y pequeñas empresas, estos movimientos en la economía, 
generan un impacto positivo, desde varias aristas, en las familias y por ende en las comunidades. Por consiguiente, éste representa un nuevo nicho de mercado para las instituciones financieras que buscan aumentar su cartera de pequeñas empresas.

En general, el poder abrir una cuenta, generar ahorro y guardar el dinero fuera del hogar de manera segura, es sin duda un factor de control sobre las finanzas. La disponibilidad crediticia y el apoyo de los diferentes entes financieros brindan un aporte incalculable para el empoderamiento productivo. En este sentido, se puede ver que en la región Latinoamericana la tasa de bancarización femenina en promedio es del $45 \%$ versus el masculino del $53 \%$, notándose además que en toda la región la bancarización masculina es, excepto por Argentina, siempre mayor (Banco Mundial , 2018). De acuerdo al mismo estudio apenas el 11\% de las mujeres adultas tienen un préstamo en una institución financiera formal y el $15 \%$ adquiere financiamiento a través de familiares o amigos. En caso de emergencia el $35 \%$ de las mujeres acuden a familiares y amigos como fuente de fondeo y solo el $9 \%$ acude al sistema financiero formal. Esto permite inferir: a) que la confianza en la banca es débil en comparación a fuentes cercanas como son amigos y familiares, sin embargo, no siempre existe apertura por pate de los miembros cercanos, o disponibilidad en ahorros para satisfacer las necesidades y urgencias de sus seres queridos, lo cual en muchas ocasiones puede lograr un vacío financiero difícil de solventar; o, b) que los productos en términos de servicios brindados por parte de los bancos no son de fácil acceso o cuentan con altas barreras, lo que restringe la llegada óptima a quienes más lo requieren.

Los servicios financieros internacionales (DFS) por sus siglas en inglés, representan una alternativa viable dentro de las estructuras bancarias para proporcionar herramientas de acceso en poblaciones de bajos recursos, a través de plataformas de dinero móvil y servicios bajo la utilización de teléfonos móviles (Mustafa , y otros, 2019). El acceso a un celular, para transacciones financieras e información de mercados, operaciones financieras, recordatorios para ahorrar, información de precios y empleos, es el mecanismo menos costoso y más efectivo para incrementar las inversiones comerciales en mujeres, adicional a ser una herramienta que ha sido considerada entre las intervenciones para el incremento de la productividad y los ingresos de las mujeres en países en desarrollo (Women's Economic Empowerment, 2018).

La disponibilidad del crédito es uno de los pilares sobre los que se sustenta la economía, permite acceder a capital de trabajo y atender las necesidades económicas que tiene la sociedad para incrementar la productividad (Banco Mundial , 2018). En América Latina y el Caribe, la inserción de las mujeres al sistema financiero ha estado marcado por una mayor participación o acceso a los productos financieros de las Instituciones Micro financieras, quienes han favorecido la inclusión financiera de mujeres dado que los resultados que han logrado han sido de mayor acceso a la propiedad o titularidad de sus negocios (CAF, 2018). Es importante tener en cuenta que la inclusión financiera es más que acceso a crédito. El crecimiento inclusivo también significa un mejor acceso a productos de ahorro y mitigación de riesgos. Esto significa que, los que fueron marginados anteriormente, también tendrán acceso a una infraestructura financiera que funcione bien y que permita a las personas y empresas participar de manera más activa y ecuánime en la economía, al tiempo que se protegen los derechos de los usuarios. 
Si bien las IFM han facilitado el acceso por parte de las mujeres a los créditos de corto plazo, han tenido menos éxito en generar servicios que satisfagan las necesidades de capital de inversión de las mujeres el cual fomente su crecimiento y empoderamiento a largo plazo (CAF, 2018). Esto debido a que los productos entregados no cumplen con una integralidad de servicios, dejando vacíos que dificultan el proceso o que no lo permiten potencializarlo al máximo.

En la Cumbre del G-20 de Seúl en noviembre de 2010 los líderes de los países miembros respaldaron el documento "Plan de acción de Inclusión financiera" la cual presenta varias recomendaciones para fomentar el acceso a servicios financieros de las mujeres que se presenta a continuación:

- Desarrollar diagnósticos específicos por país y fomentar estrategias de inclusión de género en las agendas nacionales.

- Aumentar el acceso legal de las mujeres a la propiedad mejora el acceso a las garantías y el control sobre los activos, fortaleciendo sus incentivos y la capacidad de hacer crecer un negocio.

- Fomentar la formalización de las pequeñas empresas.

- Expandir la infraestructura financiera tales como buros crediticios y registros colaterales para reducir los costos de colocación de créditos.

- Reforzar el acceso de las PYME a pequeños tribunales de reclamos y alternativa de mecanismos de resolución de conflictos.

- Implementar mecanismos de acceso a financiamiento y entrenamiento.

- Construir acuerdos público - privado mediante redes y asociaciones de mujeres para desarrollar participación política.

- Liderar esfuerzos para identificar, evaluar y apoyar en la réplica de modelos exitosos para expandir los servicios financieros a mujeres empresarias (International Finance Corporation, 2018).

Un estudio realizado por (Women's Economic Empowerment, 2018) llega a una serie de lecciones empíricas que se dan en el marco del emprendimiento femenino, si bien no cuenta con evidencia en costos y beneficios reales, permite conocer y avanzar sobre los primeros pasos dados. Entre estas lecciones están:

- El capital solo, como préstamo o donación no es suficiente para fomentar el crecimiento y sostenibilidad de los negocios.

- Es importante combinar el capital con capacitación, visitas técnicas para lograr un costo eficaz.

- El transferir capital de forma no líquida, como a través de inventarios, ayuda a mejorar el buen uso del dinero.

- Los servicios financieros por medio de uso de celulares, son menos costosos y más eficientes.

- Capacitación en administración mejora las prácticas de las emprendedoras, sin embargo, su efecto es poco mesurable.

- El apoyo al punto anterior con visitas técnicas, asesoramiento personalizado y focalizado en los negocios de las mujeres, ayuda mucho más a generar mejores resultados. 
- El contar con cuentas de ahorro eleva el ingreso empresarial de las mujeres, fomentando el ahorro y la inversión en los nuevos negocios.

\section{Resultados}

Si bien los temas de inclusión financiera no siempre son topados en las mesas de diálogo de empoderamiento productivo femenino, se ha podido evidenciar como el trabajar estos asuntos de manera coordinada puede llegar a lograr un mejor resultado en ambos.

La mujer muestra ser una potencial catalizadoras del progreso de un país, siempre y cuando los temas que le generan especial atención, por sobre todo su enfoque a la familia, estén bien atendidos, por lo que el trabajo por parte de las instituciones financieras para la creación de productos direccionados específicamente al fomento productivo de este sector no atendido, podría mejorar tanto los índices de bancarización de mujeres, así como el del emprendedurismo por parte de ellas, contribuyendo así en el mejoramiento de la calidad de vida de sus núcleos familiares, que al multiplicarse, lograrían un mejoramiento en la situación de los estratos más bajos.

La tarea de innovar en los servicios y productos financieros con un enfoque de género es sin duda imperativa para bancos, cooperativas, cajas de créditos y ahorros, IMF y áreas del gobierno que tengan como población objetivo las personas de menores recursos, aprovechando los importantes avances logrados en la accesibilidad y la asequibilidad que brinda la telefonía móvil y los servicios financieros digitales, miles de mujeres podrán acceder a servicios formales, lo que conlleva a los beneficios mencionados anteriormente. La tecnología además podría ayudar a una correcta canalización de los fondos, para que el dinero entregado pueda direccionarse de manera correcta a los destinatarios relacionados directamente con el negocio y no a al gasto familiar.

En este sentido se puede recomendar al caso ecuatoriano, específicamente en el contexto de la economía popular y solidaria direccionada a las mujeres, el trabajo mancomunado entre quienes buscan el empoderamiento productivo y los gestores de servicios financieros mediante la generación de productos en servicios financieros específicos para dicho sector, considerando su situación como núcleos de hogar y bajo una serie de puntos integrados en dicha oferta, entre los cuales podría atarse a una serie de capacitaciones para el correcto uso de dinero que fomente la sostenibilidad de su negocio y por ende la de su hogar, el seguimiento continuo al correcto uso de los créditos, y sobre todo a medios que permitan no descuidar su situación económica familiar sin que esto interrumpa el buen funcionamiento de sus negocios.

\section{Conclusiones}

El término de inclusión financiera nace con el objetivo de disminuir la desigualdad en cuanto al acceso a servicios bancarios como son créditos, transferencias, métodos de pago y otros. Esta desigualdad no se da solo en términos económicos, sino también a nivel de género, perjudicando a las mujeres sobre todo de ingresos bajos, quienes cuentan con una serie de barreras para acceder a dichos instrumentos, como son: demanda de tiempo femenino 
relacionada con labores domésticos, falta de identificación formal, menor porcentaje de propiedad de teléfonos móviles, falta de políticas y prácticas específicas del género, entre otras.

A pesar de estos bloqueos, en aquellos casos que se ha facilitado la obtención de cuentas a mujeres cabeza de hogar, se ha demostrado que debido a su responsabilidad materna, los resultados obtenidos no solo han sido beneficiosos en temas de pagos sino que el dinero generado se ha sido utilizado en educación, alimentación, salud y otras acciones que mejoran la calidad de vida de su entorno; es relevante mencionar que un acompañamiento de educación técnica y financiera ayuda a que se potencialice dichos resultados, logrando una correlación positiva entre el empoderamiento productivo de la mujer y la calidad de vida de la población. Se ha evidenciado también que el nivel de seguridad laboral varía según el nivel de ingreso, el cual también depende del nivel de educación con el que las mujeres cuenten, lo cual perjudica a los grupos más débiles, quienes sin políticas públicas fuertes es muy complicado que puedan generar logros importantes en su empoderamiento.

El acceso a sistemas financieros formales permite aumentar la productividad que está vinculada en el desarrollo económico, mejora las oportunidades del mercado laboral y ayuda al crecimiento de las micro y pequeñas empresas, generando también un impacto positivo en las familias y comunidades.

El teléfono móvil es la herramienta más económica y muy efectiva para la generación de productos financieros accesibles y asequibles. La inserción de las mujeres al sistema financiero ha generado resultados positivos sin embargo la necesidad de ofrecer productos específicos direccionados a este género es imprescindible y urgente.

\section{Bibliografía}

Acerca de la aplicación de MapReduce + Hadoop en el tratamiento de Big Data. (s.f.).

Aguilar, D. (2014). Proyecto para la creación de una escuela de capacitación de mandos medios para el sector hotelero de la ciudad de Quito. Quito: Tesis de pregrado Universidad Politècnica Salesiana.

Allen, F., Demirgug- Kunt, A., Klapper, L., \& Martinez, M. S. (15 de agosto de 2018). The Foundations of Financial Inclusion. Obtenido de Worldbank: http://documents.worldbank.org/curated/en/348241468329061640/pdf/wps6290.pdf

Amador- Ruiz, J., \& Briones- Peñalver, A. (2017). Estudio del emprendimiento en mujeres: evidencia empírica en el muncipio de León en Nicaragua. Tourism \& Management Studies, 73- 82. doi:DOI: 10.18089/tms.2017.13308

Armas, M., \& Colmenares, M. (2009). Educación para el desarrollo de la cultura tributaria. REDHECS, 6(4), 141-160. Obtenido de https://dialnet.unirioja.es/descarga/articulo/2937210.pdf

Arriaga, G., Reyes, M., Olives, J., \& Solórzano, V. (2017). Análisis de la cultura tributaria: Impuesto a la renta par personas naturales no obligadas a llevar contabildad, Provincia de 
Santa Elena. Ciencias Pedagógicas e Innovación UPSE, 5(3), 118-127. doi:DOI http://dx.doi.org/10.26423/rcpi.v5i3.192

Asociación Internacional de Franquicias. (2012).

Banco Central del Ecuador. (2018). Cuentas Nacionales. Obtenido de https://www.bce.fin.ec/index.php/component/k2/item/763

Banco Central del Ecuador. (15 de Febrero de 2018).

https://contenido.bce.fin.ec/documentos/Estadisticas/SectorExterno/BalanzaPagos/balan zaComercial/ebc201802.pdf.

Banco Mundial . (19 de julio de 2018). Banco Mundial, La Inclusión financiera es un factor clave para reducir la pobreza e impulsar la prosperidad. . Obtenido de https://www.bancomundial.org/es/topic/financialinclusion/overview

Banco Mundial . (20 de julio de 2018). Gender at work, un complemento del Informe sobre el desarrollo mundial relativo al empleo. Obtenido de http://www.bancomundial.org/es/topic/gender/publication/gender-at-work-companionreport-to-world-development-report-2013-jobs

Bedoya, A., \& Vásconez, B. (2010). Entendiendo la moral tributaria en Ecuador. Revista Fiscalidad(5), 91-132. Obtenido de https://cef.sri.gob.ec/pluginfile.php/16836/mod_page/content/64/5_3.pdf

Berlanga, V., Rubio, M., \& Vilá, R. (2013). Cómo aplicar árboles de decisión en SPSS. Revista d'Innovació i Recerca en Educació, 6(1), 65-79. Obtenido de http://diposit.ub.edu/dspace/bitstream/2445/43762/1/618361.pdf

BID. (21 de julio de 2018 ). Empoderamiento económico de las mujeres . Obtenido de http://amja.org.ar/wpcontent/uploads/2017/03/Empoderamiento_Economico_de_las_Mujeres.pdf

Briones, R. A. (03 de 2018). La Política Comercial y las Importaciones de Bienes de Consumo en Ecuador. período 2010-2016. Obtenido de http://repositorio.ug.edu.ec/browse?type=author\&value=Briones+Mora\%2C+Raquel $+\mathrm{Ab}$ igail

CAF. (2018). InclusIón FInancIera de las Mujeres en América Latina. Serie PolíticaS PúblicaS y tranSformación Productiva.

Caiza, P. (2017). Sustentabilidad y el Estado constitucional de derechos intercultural y plurinacional - ecuatoriano. Quito: Flacso.

Caiza, P. (2018). Ensayo El turismo comunitario, una alternativa al desarrollo local desde la cooperación internacional. Quito: Flacso .

Caiza, P., \& Peralta, L. (2017). Turismo sostenible, desde un enfoque de sustentabilidad fuerte: Casos de estudio Yunguilla y Mindo. Quito: Flacso. 
Calle, J. (2017). Revolución Big Data en el turismo. Análisis de las nuevas fuentes de datos para la creación de conocimiento en los Destinos Patrimonio de la Humanidad de España.

Cámara de Comercio de Guayaquil. (2018). El Impacto de las Salvaguardias sobre la Poducción en el Ecuador. Departamento de Investigación y Proyectos. Recuperado el 21 de 11 de 2018, de http://www.lacamara.org/ccg/Docs\%20generales/El\%20efecto\%20de\%20las\%20salvagu ardias\%20-\%20CCG.pdf

Carrasco, M. (2010). La ciudadania fiscal: algunas reflexiones. Revista Fiscalidad(5), 11-66. Obtenido de https://cef.sri.gob.ec/pluginfile.php/16836/mod_page/content/64/5_2.pdf

Castiblanco Rozo, C. (2015). Los paradigmas de la sostenibilidad. Bogotá: Cepal. Recuperado el 16 de abril de 2019 , de https://www.cepal.org/sites/default/files/.../presentacion_carmenza_castiblanco.pdf

CEPAL. (19 de julio de 2018). Comisión Económica para América Latina. Obtenido de https://www.cepal.org/sites/default/files/news/files/inclusion_financiera_para_las_mujere s.pdf

Cuentas Nacionales. (2018). Banco Central del Ecuador. Obtenido de https://www.bce.fin.ec/index.php/component/k2/item/763

Dastourian, B., Kawamorita, H., Seyyed Amiri, N., \& Moradi, S. (2017). Women entrepreneurship: effect of social capital, innovation and market knowledge. AD Minister, 115-130.

De la Paz, M. (2018). Torniquete a la salida de dólares.

Dominguez A., Y. A. (2014). Acerca de la aplicación de MapReduce + Hadoop en el tratamiento de Big Data. La Habana: Revista Cubana de Ciencias Informáticas.

Durán, J., \& Pellandra, A. (2017). La Irrupción de China y su Impacto sobre la Estructura Productiva y Comercial en América Latina y el Caribe. Naciones Unidas, Santiago.: ISSN 1680-869X.

El Comercio. (19 de mayo de 2017). El big data le haxe un zoom al consumidor. El Comercio.

El economista. (21 de agosto de 2017). América Economia. Obtenido de Estas son las 10 cadenas hoteleras más grandes del mundo: https://lifestyle.americaeconomia.com/articulos/estasson-las-10-cadenas-hoteleras-mas-grandes-del-mundo

El telegrafo. (06 de diciembre de 2016). Quito, puntal económico de Ecuador. La ciudad cumple 482 años con dos desafíos pendientes.

Ellis, E. (2018). Hacia una Asociación Estratégica: Las Inversiones de China en América Latina. . Revista de Red CAEM., 27.

Española, R. A. (2017). Diccionario de la lengua española. 
Evans, P. (2014). De la deconstrucción a los Big Data. BBVA.

Examen Estadístico del Comercio Mundial 2016. (16 de 10 de 2019). El Comercio Mundial en 2015 - 2016. Obtenido de https://www.wto.org/spanish/res_s/statis_s/wts2016_s/WTO_Chapter_03_s.pdf

Federación Hotelera del Ecuador. (09 de Julio de 2019). Guía de Hoteles. Obtenido de https://www.hotelesecuador.com.ec

Feijoó, W. E. (04 de 2019). Impacto de las salvaguardias en la balanza de pagos en Ecuador, período 2015 - 2017. Universidad de Guayaquil, Facultad de Ciencias Económicas, Carrera de Economía. Guayaquil. Obtenido de http://repositorio.ug.edu.ec/bitstream/redug/40493/1/TFEIJOO\%20DURAZNO\%20WLADIMIR\%20ELIAS.pdf

Fernández, M., \& Calero, J. (2011). Los efectos no monetarios de la educación, Análisis a partir del consumo de los hogares. Revista de Educación, 419-442. Obtenido de http://www.revistaeducacion.mec.es/re355/re355_18.pdf

Fundación de Microfinanzas BBVA. (21 de julio de 2018). Empoderamiento de la mujer. Obtenido de http://www.fundacionmicrofinanzasbbva.org/desarrollo-delsector/empoderamiento-la-mujer/

Galimany. (2014). La creación de valor en las empresas a través del Big Data. 33.

Garcia, Y., Jimenez, M., \& Escamilla, S. (2018). Emprendimientos Femeninos en Ecuador. Revista Publicando, 57- 66.

Gartner. (2016). IT Glossary. Obtenido de https://www.gartner.com/it-glossary/big-data.

Gobierno Provincial de Pichincha. (2015). Plan de Desarrollo y Ordenamiento Territorial Pichincha 2015-2019. Quito: Consejo Provincial de Pichincha.

Hernández, R., Fernández, C., \& Baptista, M. d. (2010). Metodología de la investigación, (ISBN: 978-607-15-0291-9) (Quinta. ed.). México.

Holloway, K., Niazi, Z., \& Rouse, R. (20 de julio de 2018). Poverty-Action. Obtenido de https://www.poverty-action.org/sites/default/files/publications/Womens-EconomicEmpowerment-Through-Financial-Inclusion-Final-Spanish-Web.pdf

INEC. (2018). Empresas de Ecuador: Quito lidera en cantidad. Obtenido de elemprendedor.ec: http://www.elemprendedor.ec/quito-lider-empresas-de-ecuador/

International Finance Corporation. (15 de agosto de 2018). Strengthening access to finance for Women- Owned SME in Developing Countries. Obtenido de https://www.ifc.org/wps/wcm/connect/a4774a004a3f66539f0f9f8969adcc27/G20_Wome n_Report.pdf?MOD=AJPERES

Iranzo, J. E. (2012). La demanda de turismo. revista de Economía Mundial, 127-149. 
Jiménez , H., Andrade, V., Sánchez, S., Bustos, P., Quishpe, J., \& Flores, S. (01 de Octubre de 2017). Ministerio de Comercio Exterior. Obtenido de https://www.comercioexterior.gob.ec/wp-content/uploads/2017/11/Bolet\%C3\%ADnMensual.pdf

Jimenez, A. d. (2008). Las cadenas hoteleras en el mundo y evolución de su operación en México al inicio del siglo XXI . Innovar.

La Hora. (18 de 07 de 2011). Comercio: Puntos Claves para Exportar a China. Obtenido de https://lahora.com.ec/noticia/1101174682/comercio-puntos-claves-para-exportar-a-china

Lasio, V., Ordeñana, X., Caicedo, G., Samaniego, A., \& Izquierdo, E. (2017). Global Entrepreneurship Monitor. Guayaquil: ESPAE- ESPOL.

Leff, E. (2000). Globalización, ambiente y sustentabilidad del desarrollo. En E. Leff, Saber ambiental: sustentabilidad, racionalidad, complejidad, poder (págs. 17-30). México: Siglo XXI.

Lopez, C. (2012). Efecto de la educación sobre el delito: evidencia para Argentina. (Tesis de maestría, Universidad Nacional de La Plata). Obtenido de http://sedici.unlp.edu.ar/bitstream/handle/10915/24608/Documento_completo_.pdf?sequ ence $=1$

López, F. (2012). Incidencia de los conocimientos sobre tributación y el cumplimiento de las obligaciones tributarias entre el pequeño comerciante del Barrio el Beaterio en el Sector de Guajaló. (Trabajo de grado). Universal Central del Ecuador, Quito, Ecuador.

Maldonado J., S. S. (2015). El Big Data como ventaja competitiva. Querétaro, México: Universidad Autónoma de Querétaro.

Maldonado J., S. S. (2015). El Big Data como ventaja competitiva. Querétaro, México: Universidad Autónoma de Querétaro.

Martinez, J. M. (2015). "Big Data"; aplicación y utilidad para el sistema sanitario. FARMACIA HOSPITALARIA, ISSN: 2171-8695., 1.

Mazo, E. (2014). China es ya la Primera Potencia Mundial. Expansión.com.

Mckinsey Global, I. (2016). The Age of Analytics: Competing in a Data-Driven World.

Melgar, J. (2000). El nichologo y los nichos de mercado como nuevos elementos de mercadeo en el siglo XXI. Theorethikos, año III, número 3, julio-septiembre.

Mendoza, F., Palomino, R., Robles, J., \& Ramírez, S. (2016). Correlación entre cultrua tributaria y educación tributaria universitaria: Caso Unversidad Estatal de Sonora. Revista Global de Negocios, 4(1), 61-76. Obtenido de ftp://ftp.repec.org/opt/ReDIF/RePEc/ibf/rgnego/rgn-v4n1-2016/RGN-V4N1-2016-5.pdf 
MIES. (15 de agosto de 2018). Ministerio de Economía Popular y Solidaria. Obtenido de Acceso a Financiemiento capital y propiedad: https://www.inclusion.gob.ec/1-acceso-afinanciamiento-capital-y-propiedad-2/

Ministerio de Comercio Exterior e Inversión. (Noviembre de 2017). Obtenido de https://www.comercioexterior.gob.ec/wp-content/uploads/2018/01/Informe-MensualDiciembre_4.pdf

Ministerio de Comercio Exterior e Inversión. (15 de 11 de 2018). Salvaguardia por Balanza de Pagos. Obtenido de https://www.comercioexterior.gob.ec/boletin-de-prensasalvaguardia-por-balanza-de-pagos/

Ministerio de Comercio Exterior e Inversiones. Informe Mensual de Comercio Exterior. (Noviembre de 2017). Obtenido de https://www.comercioexterior.gob.ec/wpcontent/uploads/2018/01/Informe-Mensual-Diciembre_4.pdf

Ministerio de Economía y Finanzas. (2017). Cifras del Presupuesto General del Estado 2018. Obtenido de https://www.finanzas.gob.ec/wpcontent/uploads/downloads/2017/11/Proforma_2018_para_Asamblea.pdf

Mintur. (2016). Reglamento de Alojamiento Turístico. Quito: Ministerio de Turismo.

Mora, G. (Octubre de 2011). Las Importaciones de Calzado desde La República Popular China hacia el Ecuador Periodo 2009 - 2010. Universidad de Guayaquil. Facultad de Ciencias Económicas. Tesis para obtener el Título de Economista. Obtenido de http://repositorio.ug.edu.ec/bitstream/redug/2352/1/Mora\%20Orodo\%C3\%B1ez\%20Gen aro\%20Bolivar.pdf

Morales, N., Méndez, M., \& Aguilera, O. (2005). Cultura tributaria y conribuyentes: datos y aspectos metodológicos. Fermentum. Revista Venezolana de Sociología y Antropología, 15(44), 332-352. Obtenido de http://www.redalyc.org/articulo.oa?id=70504404

Moreno, C. (2015). Empoderamiento económico y género: Un caso de implementación de política en el ámbito municipal . Gestión y Política Pública, 579- 612.

Mustafa, M., Mazhar, N., Asghar, A., Zafar, M., Razaq, L., \& Anderson, R. (2019). Digital Financial Needs of Micro- entrepreneur Women in Pakistan: Is Mobile Money the Answer? CHI Conference on Human Factors in Computing Systems Proceedings, 1- 12. doi:https://doi.org/10.1145/ 3290605.3300490

Naciones Unidas. (20 de julio de 2018). Resolución aprobada por la Asamblea General. Obtenido de http://www.un.org/es/comun/docs/?symbol=A/RES/70/1

ONU MUJERES. (1 de agosto de 2018). Empoderamiento económico. Obtenido de http://ecuador.unwomen.org/es/que-hacemos/empoderamiento-economico

Panam Post. (20 de Junio de 2017). Colombia es el país más peligroso para turistas según Foro Económico Mundial. Panam Post. 
Peña, H. (10 de Noviembre de 2018). Cámara de Comercio de Guayaquil. Relación Comercial Ecuador - China. Obtenido de http://www.lacamara.org/websiteantes/images/boletines/informacion-comercial/relacion\%20comercial\%20ecuador\%20$\% 20$ china.pdf

Peña, H. (15 de 10 de 2019). Relación Comercial ECUADOR - CHINA. Obtenido de http://www.lacamara.org/website-antes/images/boletines/informacioncomercial/relacion\%20comercial\%20ecuador\%20-\%20china.pdf

Pérez, G. D., Puig, M. Z., \& Fernández, R. M. (julio - diciembre 2016). La expansión internacional de la industria hotelera de los países desarrollados como opción estratégica para los países subdesarrollados. Economía y Desarrollo, vol 157 N.2.

Pérez, G., Pineda, U., \& Arango, M. (2011). La capacitación a través de algunas teorías de aprendizaje y su influencia en la gestión de la empresa. Revista Virtual Universidad Católica del Norte(33), 1-22.

Pérez, P., \& Souza, A. (2013). Como quantificar os aspectos qualitativos nas escalas de classificação de resultados NOC para etiquetas psicossocioculturais. Revista da Escola de Enfermagem da USP, 47(3), 728-735. doi:DOI: 10.1590/S0080-623420130000300029

Perrotti, D. (2015). La República Popular de China y América Latina: Impacto del Crecimiento Económico Chino en las Exportaciones Latinoamericanas. Revista Cepal 116. Agosto 2015.

Quintanilla, J. (2012). La universidad en la cultura tributaria. RETOS. Revista de Ciencias de la Administración y Economía, 2(3), 105-114. Obtenido de http://www.redalyc.org/pdf/5045/504550953007.pdf

Ramírez, R. (2014). El "Sueño Chino" de XI JINPING. La "Quinta Generación de Dirigentes" y su Programa de Gobierno. (I. 2386-2491, Ed.) La Albolafia: Revista de Huamnidades y Cultura.

Revista Líderes. (10 de 11 de 2018). Obtenido de https://www.revistalideres.ec/lideres/productos-ecuatorianos-demanda-chinamariella.html

Rivadeneira, E. V., \& León, N. M. (2017). Precipitaciones extremas en la ciudad de Quito, provincia de Pichincha- Ecuador . Ingeniería Hidráulica y Ambiental, Recuperado en 13 de mayo de 2019, de http://scielo.sld.cu/scielo.php?script=sci_arttext\&pid=S168003382017000200008\&lng=es\&tlng=es. .

Roa, M. J. (2013). Inclusión Financiera en América Latina y el Caribe: acceso, uso y calidad. . Boletín CEMLA, 121.

Rodríguez Rojas, P. (2003). La andragogía y el constructivismo en la sociedad del conocimiento. Laurus, 9(15), 80-89. 
Rodriguez, J. M., \& otros. (2012). Influencia de los principios organizativos, parámetros de diseño y factores de contingencia en las estructuras organizativas de las cadenas hoteleras españolas y mexicanas. España: vision libros.

San Vicente Portes, L., Atal, V., \& Juárez, M. (2019). From households to national statistics. Economic Moldelling, 286- 294. doi:https://doi.org/10.1016/j.econmod.2019.01.024

Sarmiento, B. (2014). La cultura tributaria en el Ecuador. Observatorio de la economía Latinoamericana(201). Obtenido de http://www.eumed.net/cursecon/ecolat/ec/2014/cultura-tributaria.html

Sistema Institucional de Indicadores Turisticos. (2017).

Sistema Institucional de Indicadores Turísticos SIIT. (25 de Abril de 2019). Datos de EcuadorQuito.

Soto, M. (2016). La cultura tributaria: una contribución para la construcción colectiva del desarrollo sustentable. Novum Scientiarum, 2(1), 46-56. Obtenido de http://www.ecoambienteydesarrollo.org/revista/ojs/index.php/novum/article/view/62/cult uratributaria

SRI. (2018). Estadísitcas generales de recaudación. Obtenido de http://www.sri.gob.ec/web/guest/estadisticas-generales-derecaudacion?p_auth=KeAzH2ym\&p_p_id=busquedaEstadisticas_WAR_BibliotecaPortle t_INSTANCE_EVo6\&p_p_lifecycle=1\&p_p_state=normal\&p_p_mode=view\&p_p_col_ id=column-1\&p_p_col_count=2\&_busquedaEstadisticas_

Teinteresa. (2018). Qué tipo de comprador eres? Obtenido de teinteresa.es: http://www.teinteresa.es/espana/tipo-comprador_0_1049295310.html

Terán, N. C. (Julio de 2018). Análisis de los Efectos del Comercio Bilateral Ecuador - China en los Indicadores Macroeconómicos PIB, Balanza Comercial y Empleo del Ecuador, durante el perìodo 2013-2017. Quito.

Torres, M., Fermín, Y., Arroyo, C., \& Piñero, M. (2000). La horizontalidad y la participación en la Andragogía. Educere, 4(10), 25-34.

UCACUE. (2016). Acuerdo de cooperación interinstitucional entre la UCACUE y el SRI. [Acta convenio]. Archivo UACUE.

Valencia, D. (2015). Ministerio de Comercio Exterior. Obtenido de https://www.comercioexterior.gob.ec/wp-content/uploads/downloads/2016/04/Informede-Gestion-Institucional-MCE-2015.pdf

Valls, J. F. (2017). Big Data, Atrapando al consumidor. Barcelona, España: Profit.

Vazquez, M., Camacho, G., \& Garcia, C. (2016). EMPODERAMIENTO DE LA MUJER A TRAVÉS DE INNOVACIÓN SOCIAL EN EMPRESAS SOCIALES INDÍGENAS.

Esta obra se comparte bajo la licencia Creative Common Atribución-No Comercial 4.0 International (CC BY-NC 4.0) Revista de la Universidad Internacional del Ecuador. URL: https://www.uide.edu.ec/ 
Revista de Administração Mackenzie, 164- 190. doi:http://dx.doi.org/10.1590/167869712016/administracao.v17n6p164-190

Women's Economic Empowerment. (7 de Agosto de 2018). A Roadmap for promoting women's Economic Empowerment. Obtenido de http://www.womeneconroadmap.org/roadmap

World Economic Forum . (2018). El Foro Económico Mundial. Obtenido de El Big Data y los consumidores | Foro Económico Mundial: https://es.weforum.org/agenda/2017/12/el-bigdata-y-los-consumidores

Zalles, J. (2016). El gallito de la peña: turismo, uso de suelo y conservación biológica en el noroccidente de Pichincha, Ecuador. Quito.

Zambrano, R. (2005). Quito: Patrimonio de la Humanidad. Quito: Libresa.

Zurita, A. (4 de Marzo de 2016). Instituto Español de Estudios Estratégicos. China: El Despertar del Dragon Rojo. Obtenido de http://www.ieee.es/Galerias/fichero/docs_marco/2016/DIEEEM032016_China_Despertar_DragonRojo_AlfonsoZurita.pdf 